\title{
NEUROSCIENCE
}

\section{Unbiased, whole-brain imaging of neural circuits}

Functional ultrasound imaging enables unbiased identification of behaviorally relevant brain regions across the whole brain.

$\mathrm{N}$ eural circuits are typically analyzed via optical means with limited fields of view, which makes it difficult to obtain a system-wide understanding of behaviorally evoked neural activity. Functional magnetic resonance imaging is an alternative for unbiased imaging of neural circuits, but the spatial resolution of this technology is limited.

Botond Roska from the Friedrich Miescher Institute for Biomedical Research in Basel, postdoc Émilie Macé, and their colleagues used functional ultrasound imaging in awake, head-fixed mice to identify brain regions in which activity was evoked by the optokinetic reflex, a stabilizing eye motion induced by image drift on the retina. With a resolution of about $100 \mu \mathrm{m}$, the technology makes it possible to monitor hemodynamic activity as a proxy for neural activity even in small nuclei in the brain.

Currently, ultrasound probes can image activity in a single plane, and the researchers achieved full-brain coverage by stepping the probe across the brain. To obtain sufficient trial repetitions and achieve a high signal-to-noise ratio, the researchers opted to image each plane for 20 minutes, requiring a total of 6.5 hours for each brain. In principle, it is feasible to image faster, with trade-offs in the signal-tonoise ratio and the stimulus repetitions.

The functional ultrasound approach allowed the researchers to visualize activity in 87 brain regions during the presentation of drifting stimuli. In these experiments, neural activity can be caused by the processing of retinal signals, the transformation of those signals into motor commands, or eye motion. The researchers could distinguish such activity by imaging brain activity in mice that exhibited nystagmus (uncontrolled eye movements) or that could not move their eyes. The researchers determined that 12 of the 87 brain regions that exhibited activity functioned in the reflex pathway proper.

While this study elegantly illustrates the power of functional ultrasound imaging, developments that enable truly volumetric ultrasound imaging would be desirable to speed up data acquisition.

\section{Nina Vogt}

Published online: 30 January 2019

https://doi.org/10.1038/s41592-019-0313-2

Research papers

Macé, É. et al. Whole-brain functional ultrasound imaging reveals brain modules for visuomotor integration. Neuron 100, 1241-1251 (2018).

\section{We Create Solutions}

Ultra Precise Motion Control - D.C. Servo motors down to $20 \mathrm{~nm}$, piezos down to $1 \mathrm{~nm}$, and low drift XYZ stages.

Microscopy - Automation, modular microscopes, autofocus complete light sheet systems, and components.

OEM - Custom designed systems to user specifications.

www.asiimaging.com

info@asiimaging.com

(800) $706-2284$ or (541) 461-8181

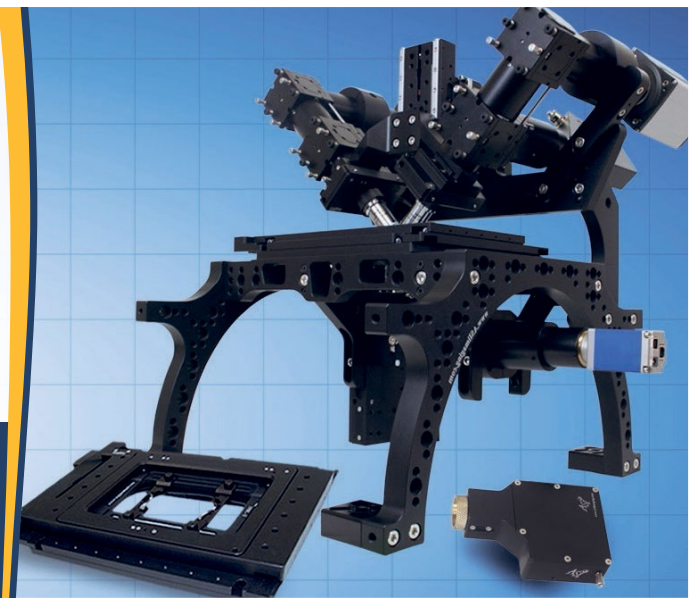

